\title{
Teenage-onset progressive myoclonic epilepsy due to a familial C9orf72 repeat expansion
}

Jelle van den Ameele MD $\mathrm{PhD}^{1,2^{*}}$, Ivana Jedlickova $\mathrm{MSc}^{3^{*}}$, Anna Pristoupilova $\mathrm{MSc}^{3}$, Anne Sieben MD ${ }^{1,4,5}$, Sara Van Mossevelde $\mathrm{MD}^{5,6}$, Chantal Ceuterick-de Groote $\mathrm{PhD}^{7}$, Helena Hůlková MD PhD ${ }^{3,8}$, Radoslav Matej MD $\mathrm{PhD}^{8,9}$, Alfred Meurs MD PhD ${ }^{1}$, Christine Van Broeckhoven PhD DSc ${ }^{5,6}$, Samuel F Berkovic MD ${ }^{10}$, Patrick Santens MD PhD ${ }^{1}$, Stanislav Kmoch $\mathrm{PhD}^{3}$, Bart Dermaut MD PhD ${ }^{1,11,12}$

1. Department of Neurology, Ghent University Hospital, Ghent, Belgium.

2. Current address: Department of Clinical Neurosciences and WT/CRUK Gurdon Institute, University of Cambridge, Cambridge, UK.

3. Institute for Inherited Metabolic Disorders, Prague, First Faculty of Medicine, Charles University in Prague, 12000 Prague, Czech Republic.

4. Neurodegenerative Brain Diseases Group, Center for Molecular Neurology, VIB, Antwerp, Belgium.

5. Neuropathology and Laboratory of Neurochemistry and Behavior, Institute Born-Bunge, University of Antwerp, Antwerp, Belgium.

6. Laboratory of Neurogenetics, Institute Born-Bunge, University of Antwerp, Antwerp, Belgium

7. Laboratory of Neuromuscular Pathology, Institute Born-Bunge and Translational Neurosciences, University of Antwerp, Belgium.

8. Institute of Pathology, First Faculty of Medicine, Charles University and General University Hospital, Prague, Czech Republic. 
9. Department of Pathology and Molecular Medicine, National Reference Laboratory for Diagnostics of Human Prion Diseases, Thomayer Hospital, Prague, Czech Republic. 10. Epilepsy Research Centre, Department of Medicine, University of Melbourne, Austin Health, Heidelberg, Australia.

11. Center for Medical Genetics, Ghent University Hospital, Ghent, Belgium.

12. Inserm U1167, Laboratoire d'Excellence Distalz, Institut Pasteur de Lille, Longevity Research Center, Université de Lille, Lille, France.

*These authors contributed equally to the manuscript.

Correspondence to: Jelle van den Ameele, WT/CRUK Gurdon Institute, Tennis Court Road, Cambridge CB2 1QN, UK. Tel +44 (0)1223 334088, Fax +44 (0)1223 334089, E-mail jv361@cam.ac.uk or Bart Dermaut, Center for Medical Genetics Gent, University Hospital Ghent, De Pintelaan 185, 9000 Ghent, Belgium. Tel +32 (0)9 33236 03, Fax +32 (0)9 332 4970, E-mail: bart.dermaut@ugent.be

\section{Author e-mails:}

jv361@cam.ac.uk; ivana.jedlickova@lf1.cuni.cz; anna.pristoupilova@lf1.cuni.cz; annesieben@yahoo.com; sara.vanmossevelde@molgen.vib-ua.be; chantal.degroote@uantwerpen.be; helena.hulkova@lf1.cuni.cz; radoslav.matej@ftn.cz; alfred.meurs@ugent.be; christine.vanbroeckhoven@molgen.vib-ua.be; s.berkovic@unimelb.edu.au; patrick.santens@ugent.be; skmoch@1f1.cuni.cz; bart.dermaut@uzgent.be 


\section{Supplemental data:}

- Supplemental information: Family history; Materials and methods.

- 2 Supplementary figures, 3 Supplementary tables.

Word count: Abstract 186; Main text 1511

Title character count: 87

References: 21

Figures: 2

Neurology search terms: [25] Cognitive disorders/dementia; [60] Epilepsy/seizures; [91]

Genetics; [173] Myoclonus 


\section{Author contributions:}

- Jelle van den Ameele: study concept and design, acquisition of data, analysis and interpretation, writing of the manuscript

- Ivana Jedlickova: study concept and design, acquisition of data, analysis and interpretation, writing of the manuscript

- Anna Pristoupilova: NGS data analysis and interpretation

- Anne Sieben: acquisition of neuropathology data, critical revision of the manuscript

- Sara Van Mossevelde: acquisition of data, critical revision of the manuscript

- Chantal Ceuterick-de Groote: electron microscopy data acquisition, analysis and interpretation, critical revision of the manuscript

- Helena Hůlková: acquisition of data, analysis and interpretation, critical revision of the manuscript

- Radoslav Matej: acquisition of data, analysis and interpretation, critical revision of the manuscript

- Alfred Meurs: EEG analysis and interpretation, critical revision of the manuscript

- Christine Van Broeckhoven: acquisition of data, analysis and interpretation, critical revision of the manuscript

- Samuel F Berkovic: study supervision, acquisition of data, analysis and interpretation, critical revision of the manuscript

- Patrick Santens: study concept and design, study supervision, acquisition of data, analysis and interpretation, writing of the manuscript

- Stanislav Kmoch: study concept and design, study supervision, acquisition of data, analysis and interpretation, writing of the manuscript

- Bart Dermaut: study concept and design, study supervision, acquisition of data, analysis and interpretation, writing of the manuscript 


\section{Disclosures:}

- Dr. van den Ameele reports no disclosures

- Ms. Jedlickova reports no disclosures

- Ms. Pristoupilova reports no disclosures

- Dr. Sieben reports no disclosures

- Dr. Van Mossevelde reports no disclosures

- Dr. Ceuterick-de Groote reports no disclosures

- Dr. Hůlková reports no disclosures

- Dr. Matej reports no disclosures

- Dr. Meurs received honoraria from serving on the advisory board of UCB and GSK and received speaker's fees and funding for a trip from UCB.

- Dr. Van Broeckhoven reports no disclosures

- Dr. Berkovic reports no disclosures

- Dr. Santens reports no disclosures

- Dr. Kmoch reports no disclosures

- Dr. Dermaut reports no disclosures 


\section{Acknowledgements}

We thank the patients and family members for their participation. I.J., A.P. and S.K. are supported by the grants 269615 from the Charles University Grant Agency and 14-36804G from the Grant Agency of the Czech Republic. Institutional support was provided by Charles University institutional programs PRVOUK-P24/LF1/3, UNCE 204011 and SVV2017/ 260367, and by the project LQ1604 NPU II from the Ministry of Education, Youth and Sports of the Czech Republic. R.M. and H.H. are supported by project of the Ministry of Health, Czech Republic (Conceptual development of research organization 64165, General University Hospital in Prague) and by Charles University (Project Progres Q28/LF1). C.V.B is supported by the Interuniversity Attraction Poles program P7/16 of the Belgian Science Policy Office (BELSPO), the Flemish initiated Impulse Program on Networks for Dementia Research (VIND), and the Flemish government initiated Methusalem Excellence Program. B.D. is supported by the Interuniversity Attraction Poles program P7/16 of the Belgian Science Policy Office (BELSPO), Inserm, the Université de Lille, the Fondation Plan Alzheimer, the LABEX (laboratory of excellence program investment for the future) DISTALZ grant (Development of Innovative Strategies for a Transdisciplinary approach to ALZheimer's disease) and INSTALZ, an EU Joint Programme - Neurodegenerative Disease Research (JPND) project. The INSTALZ project is supported through the following funding organizations under the aegis of JPND (Belgium, Research Foundation Flanders; Denmark, Innovation Fund Denmark; France, Agence Nationale de la Recherche; Sweden, Swedish Research Council; United Kingdom, Medical Research Council). The project has received funding from the European Union's Horizon 2020 research and innovation programme under grant agreement No 643417. We thank the Genomic facility in Motol University Hospital in Prague (OPPK.CZ.2.16/3.100/24022) and The National Center for Medical Genomics (LM2015091) for their instrumental and technical support with exome sequencing and data 
analyses. We thank Marleen Praet, Martin Lammens, Elfride De Baere, Kathleen Claes, Isabelle Leber, Anne De Septenville, Celine Bellenguez, Marleen Van den Broeck, JeanJacques Martin, John Staropoli and Sara Mole for their help and suggestions. 


\begin{abstract}
Background - The progressive myoclonic epilepsies (PME) are a heterogeneous group of disorders in which a specific diagnosis cannot be made in a subset of patients, despite exhaustive investigation. $C 9$ orf 72 repeat expansions are emerging as an important causal factor in several adult-onset neurodegenerative disorders, in particular frontotemporal lobar degeneration and amyotrophic lateral sclerosis. An association with PME has not been reported previously.

Objective - To identify the causative mutation in a Belgian family where the proband had genetically unexplained progressive myoclonic epilepsy.

Results - We report a 33-year old woman, who had epilepsy since the age of 15 and then developed progressive cognitive deterioration and multifocal myoclonus at the age of 18 . The family history suggested autosomal dominant inheritance of psychiatric disorders, epilepsy and dementia. Thorough work-up for PME including whole exome sequencing did not reveal an underlying cause, but a $C 9$ orf72 repeat expansion was found in our patient and affected relatives. Brain biopsy confirmed the presence of characteristic p62-positive neuronal cytoplasmic inclusions.
\end{abstract}

Conclusion - C9orf72 mutation analysis should be considered in patients with PME and psychiatric disorders and/or dementia, even when the onset is in late childhood or adolescence. 


\section{Introduction}

The progressive myoclonic epilepsies (PME) are a heterogeneous group of neurodegenerative diseases with variable age of onset and cause, all resulting in a progressive syndrome of myoclonic jerks and epileptic seizures, often accompanied by dementia and ataxia ${ }^{1}$. There are over 20 established genetic causes of PME with the more common ones being UnverrichtLundborg and Lafora body disease due to mutations in CSTB and EPM2A/EPM2B respectively, the neuronal ceroid lipofuscinoses (NCL), sialidoses due to neuraminidase deficiency, and mitochondrial encephalopathy with ragged red fibers (MERRF). A recent large multi-centre study could not identify an underlying cause in $28 \%$ of patients with $\mathrm{PME}^{2}$.

C9orf72 hexanucleotide repeat expansions are the main cause of genetic frontotemporal dementia (FTD) and amyotrophic lateral sclerosis (ALS). Average age of onset is in the $6^{\text {th }}$ decade, with a range between 27 and 83 years ${ }^{3,4}$. Since their first description in $2011^{5-7}$, the clinical phenotype associated with these mutations has expanded beyond the FTD/ALS spectrum. Parkinsonism and psychiatric features are well established now, and hyperkinetic or cerebellar movement disorders have been described ${ }^{8,9}$. Seizures are rarely described ${ }^{10,11}$ and PME has not been reported.

Here, we describe a patient with PME and a C9orf72 repeat expansion, with first symptoms appearing at the age of 15 . Onset in the paediatric years and the phenotype of PME broadens the C9orf72-related phenotypic spectrum and could provide an opportunity for patients with PME for whom no underlying cause has been detected.

\section{Case report}

A 33-year old woman was referred to the neurology department of a tertiary care hospital in Belgium because of progressive cognitive decline and speech difficulties. She had suffered 
from epilepsy with generalized tonic-clonic seizures and myoclonic jerks since the age of 15 but was seizure-free for several years under treatment with valproic acid, levetiracetam and clobazam. She was reportedly well before the age of 15 , with average school performance. She lived together with her partner and could perform all activities of daily life independently. Despite attempting higher education at the age of 18, she did not succeed and was currently employed in a sheltered workshop.

On clinical examination, the patient was alert and cooperative, she was well oriented in time and space. She had a score of $24 / 30$ on the mini mental state examination, with striking deficits in attention and upon executing more complex commands. Vital signs were normal. Neurological examination of the cranial nerves was normal, as were fundoscopy, sensory examination, strength and reflexes in all four limbs, with absence of clear pyramidal and frontal signs. Gait was slightly broad based. Small amplitude myoclonic jerks were noted in the arms, legs and fingers, which interfered with cerebellar testing, but there was no ataxia. She did not have tremor; muscle tone was normal. Speech was slow, with multifocal facial myoclonus both at rest and during speaking, and she displayed signs of neurogenic stuttering. Speech induced dystonic facial contractions were infrequently seen.

Neuropsychological testing three months after initial evaluation showed mostly deficits in attention and executive functions and to a lesser extent in verbal memory (Table e-1). Electroencephalography (EEG) repeatedly showed a moderate increase in slow activity in the theta and delta range, as well as occasional low amplitude, generalized spike-wave discharges with central maximum, without clear photosensitivity (Figure 1b).

The family history revealed several patients with adult-onset psychiatric or neurodegenerative disorders (Figure 1a); more details are in Supplementary text. The mother of the proband (II.2) had been hospitalized at the age of 47 because of anxiety and depression but subsequently developed epilepsy at the age of 48 and a progressive cognitive decline 
compatible with FTD which lead to her death at the age of 56. A maternal aunt (II.4) suffered from depression and memory problems. Neuropsychological testing suggested a possible FTD with onset in her mid-fifties (data not shown).

Adult-onset autosomal dominant PME was diagnosed and further examinations were focused on elucidating the underlying cause. Ophthalmologic examination was normal. Routine blood analysis (Table e-2) did not reveal abnormalities, as did testing of cerebrospinal fluids including dementia biomarkers (Table e-3). MRI of the brain revealed generalized cerebral and cerebellar atrophy, mostly in the bilateral parietal lobes (Figure 1c) with concordant relative hypometabolism on FDG-PET imaging. Skin and muscle biopsy were normal as were mitochondrial enzyme activities in muscle. Genetic testing for autosomal dominant spinocerebellar ataxias (SCA1-2-3-6-7-17), dentatorubral-pallidoluysian atrophy, Huntington disease and familial Alzheimer's disease (PSEN1-2, APP) was normal. Microarray based comparative genomic hybridization did not reveal pathologic copy number variations. Whole exome sequencing (WES) of the patient and her maternal aunt was performed in the context of the ANCL Gene Discovery Consortium ${ }^{12}$. The WES data set was filtered for variants in PME causative genes, namely KCNC1, CERS1, PRICKLE1, EPM2A, GOSR2, KCTD7, LMNB2, NHLRC1, PRDM8, CSTB, SCARB2, DNAJC5 $5^{13}$, having a minor allele frequency $<5 \%$ in the ExAC database. Using this approach, we did not detect any candidate variants in the proband. Additional searching for genetic alterations in a wider spectrum of epilepsy genes ${ }^{14}$ did not reveal any potential candidate mutations. Electron microscopy (EM) examination of a brain biopsy from the patient's right parietal lobe at the age of 34 showed some non-specific increase in lipopigment (Figure 2a), but no evidence for Lafora bodies or material diagnostic for NCL. EM analysis of the muscle biopsy of the maternal aunt (II.4), was more ambiguous and suggested presence of pathological storage material in the form of lipopigment with occasional fingerprints (Figure 2b,c), 
although insufficient to establish a definite diagnosis of adult neuronal ceroid lipofuscinosis (ANCL) or Kufs Disease. Subsequent expert review of the biopsy findings within the same Consortium excluded the diagnosis of ANCL.

Next we tested for C9orf72 repeat expansion, which was positive in both our patient and the affected aunt, as well as in two distant family members (II.6 and II.8) (Figure 1a). Shortrepeat $\mathrm{PCR}^{3}$ excluded a repeat size of less than $80 \mathrm{bp}$ in the proband and her aunt. Further analysis of the brain biopsy with light microscopy examination showed clear neuronal cytoplasmic inclusions that stained positive for $\mathrm{p} 62$, but were negative for ubiquitin, TDP-43 and its hyperphosphorylated form (Figure 2d,e). Autofluorescence was slightly increased and ANCL-related staining for SCMAS, CathD and LAMP2 were negative (Figure e-1). Additional staining for Amyloid, Tau, FUS and Alpha-synuclein did not reveal pathological changes (data not shown).

\section{Discussion}

Here, we present a patient with PME and describe a remarkable association between this clinical syndrome and $C 9$ orf72 repeat expansions. This constitutes an important finding for those patients with PME of undetermined cause ${ }^{2}$, in particular because this type of intronic mutation cannot be identified through WES, as was the case in our patient. C9orf72 repeat expansions can be found in about $0.15^{9}$ to $0.2 \%{ }^{15}$ of healthy individuals, but the absence of known PME-related defects in the WES data, and the segregation of the repeat expansion in the family, support a direct pathogenic role for the C9orf72 repeat expansion in the PME phenotype of our patient.

Because of the autosomal dominant inheritance of adult-onset neuropsychiatric symptoms in family members, we focused our initial work-up on the possibility of ANCL or Kufs disease causing PME in our patient. EM analysis of muscle tissue from the patient's maternal aunt 
also suggested an increase in pathological lipofuscin-type deposits, but a definite diagnosis of ANCL could not be confirmed by the pathologists of the ANCL Consortium ${ }^{12}$. Mining publicly accessible databases with available data on $C 9$ orf 72 status ${ }^{9,16}$ did not reveal additional cases with symptoms suggestive of PME. Analysis of the ANCL patients with autosomal dominant PME but without genetic diagnosis from the ANCL consortium did not reveal other $C 9$ orf72 repeat expansions $(n=5)$. Larger cohorts of patients with PME have not been tested so far.

This report supports the novel and emerging concept of disease anticipation in families segregating a $C 9$ orf72 repeat expansion ${ }^{17}$. Symptoms associated with $C 9$ orf72 repeat expansions typically manifest after the $4^{\text {th }} \operatorname{decade}^{5}$ as illustrated by the proband's mother (II.2, onset around 47 years) and maternal aunt (II,4, onset around 55 years). Our patient had her first epileptic seizure more than 30 years earlier at the age of 15 and quickly thereafter developed progressive cognitive decline. Short-repeat PCR did not reveal the presence of short expansions in the proband or maternal aunt, and it is not yet possible to determine the exact length of longer repeat expansions. Although many factors may contribute to the clinical variation, one can speculate that meiotic repeat instability in the mother has resulted in a further expansion of the hexanucleotide repeat in the proband resulting in the very early onset and atypical PME presentation.

It is intriguing to note that for a number of mid- to late-life inherited neurodegenerative diseases like Alzheimer disease ${ }^{18}$, Huntington disease ${ }^{19}$, neuroserpinopathy ${ }^{20}$ and DRPLA ${ }^{21}$, classically presenting as dementias, the presentation can be of a PME when family members have an early onset. C9orf72 repeat expansion can now be added to this list.

Our description of PME due to C9orf72 repeat expansion expands the phenotypic spectrum associated with this intriguing non-coding hexanucleotide repeat expansion. Testing for this 
van den Ameele et al - 14

mutation should be considered in the work-up of patients with PME, even when onset is in late childhood or adolescence. 


\section{Figure legends}

Figure 1 Pedigree, neurophysiology and brain imaging of the proband.

(A) Pedigree of the affected family. Shaded symbols denote affected family members, the proband is indicated with an arrow. Confirmed heterozygous C9orf72 repeat expansions are shown as +/-; obligate carrier status is indicated as (+/-). (B) Representative electroencephalogram of the proband with a bilateral, synchronous epileptiform spike-wave discharge highlighted in gray. (C) Axial fluid-attenuated inversion recovery brain MRI of the proband demonstrating bilateral parietal lobe atrophy.

\section{Figure 2 Electron microscopy and immunohistochemistry.}

(A) Electron microscopy of the brain cortex of patient III.2 (proband) showing intraneuronal classical lipofuscin. (B,C) Electron microscopy of the skeletal muscle biopsy of patient II.4 demonstrating presence of lipofuscin-like inclusions with fingerprints. (D,D') p62 staining in the brain cortex of patient III.2, with p62 positive cytoplasmic inclusions of different shape in some neurons and occasionally in glial cells. (E) Phospho-TDP-43 negative staining in the brain cortex. Magnifications 27000x (A), 67500x (B), 107500x (C), 4x (D,E) and 20x (D'). 


\section{References}

1. Marseille Consensus Group. Classification of progressive myoclonus epilepsies and related disorders. Ann Neurol. 1990;28:113-116.

2. Franceschetti S, Michelucci R, Canafoglia L, et al. Progressive myoclonic epilepsies Definitive and still undetermined causes. Neurology. 2014;82:405-411.

3. Gijselinck I, Cruts M, Van Broeckhoven C. The Genetics of C9orf72 Expansions. Cold Spring Harb Perspect Med. Epub 2017 Jan 27.:a026757.

4. Gijselinck I, Van Mossevelde S, van der Zee J, et al. The C9orf72 repeat size correlates with onset age of disease, DNA methylation and transcriptional downregulation of the promoter. Mol Psychiatry. 2015;21:1-13.

5. Gijselinck I, Van Langenhove T, van der Zee J, et al. A C9orf72 promoter repeat expansion in a Flanders-Belgian cohort with disorders of the frontotemporal lobar degeneration-amyotrophic lateral sclerosis spectrum: a gene identification study. Lancet Neurol. 2012;11:54-65.

6. Renton AE, Majounie E, Waite A, et al. A hexanucleotide repeat expansion in C9ORF72 is the cause of chromosome 9p21-linked ALS-FTD. Neuron. 2011;72:257268.

7. DeJesus-Hernandez M, Mackenzie IR, Boeve BF, et al. Expanded GGGGCC Hexanucleotide Repeat in Noncoding Region of C9ORF72 Causes Chromosome 9pLinked FTD and ALS. Neuron. 2011;72:245-256.

8. Rohrer JD, Isaacs AM, Mizlienska S, et al. C9orf72 expansions in frontotemporal dementia and amyotrophic lateral sclerosis. Lancet Neurol. 2015. p. 291-301.

9. Beck J, Poulter M, Hensman D, et al. Large C9orf72 hexanucleotide repeat expansions are seen in multiple neurodegenerative syndromes and are more frequent than expected in the UK population. Am J Hum Genet. 2013;92:345-353. 
10. Vatsavayai SC, Yoon SJ, Gardner RC, et al. Timing and significance of pathological features in C9orf72 expansion-associated frontotemporal dementia. Brain. 2016;139:3202-3216.

11. Capasso M, Anzellotti F, Di Giacomo R, Onofrj M. Epilepsy and electroencephalographic abnormalities in $\mathrm{C} 9$ orf72 repeat expansion. Amyotroph Lateral Scler Frontotemporal Degener. 2017;18:140-141.

12. Berkovic SF, Staropoli JF, Carpenter S, et al. Diagnosis and misdiagnosis of adult neuronal ceroid lipofuscinosis (Kufs disease). Neurology. 2016;87:579-584.

13. Nosková L, Stránecký V, Hartmannová H, et al. Mutations in DNAJC5, encoding cysteine-string protein alpha, cause autosomal-dominant adult-onset neuronal ceroid lipofuscinosis. Am J Hum Genet. 2011;89:241-252.

14. Wang J, Lin Z-J, Liu L, et al. Epilepsy-associated genes. Seizure. 2017;44:11-20.

15. Majounie E, Renton AE, Mok K, et al. Frequency of the C9orf72 hexanucleotide repeat expansion in patients with amyotrophic lateral sclerosis and frontotemporal dementia: A cross-sectional study. Lancet Neurol. 2012;11:323-330.

16. Keogh MJ, Wei W, Wilson I, et al. Genetic compendium of 1511 human brains available through the UK Medical Research Council Brain Banks Network Resource. Genome Res. 2017;27:165-173.

17. Van Mossevelde S, van der Zee J, Gijselinck I, et al. Clinical Evidence of Disease Anticipation in Families Segregating a C9orf72 Repeat Expansion. JAMA Neurol. Epub 2017 Feb 13.

18. Melanson M, Nalbantoglu J, Berkovic S, et al. Progressive myoclonus epilepsy in young adults with neuropathologic features of Alzheimer's disease. Neurology. 1997;49:1732-1733.

19. Gambardella A, Muglia M, Labate A, et al. Juvenile Huntington's disease presenting 
as progressive myoclonic epilepsy. Neurology 2001. p. 708-711.

20. Davis RL, Shrimpton AE, Carrell RW, et al. Association between conformational mutations in neuroserpin and onset and severity of dementia. Lancet. 2002;359:22422247.

21. Iizuka R, Hirayama K, Maehara KA. Dentato-rubro-pallido-luysian atrophy: a clinicopathological study. J Neurol Neurosurg Psychiatry. 1984;47:1288-1298. 


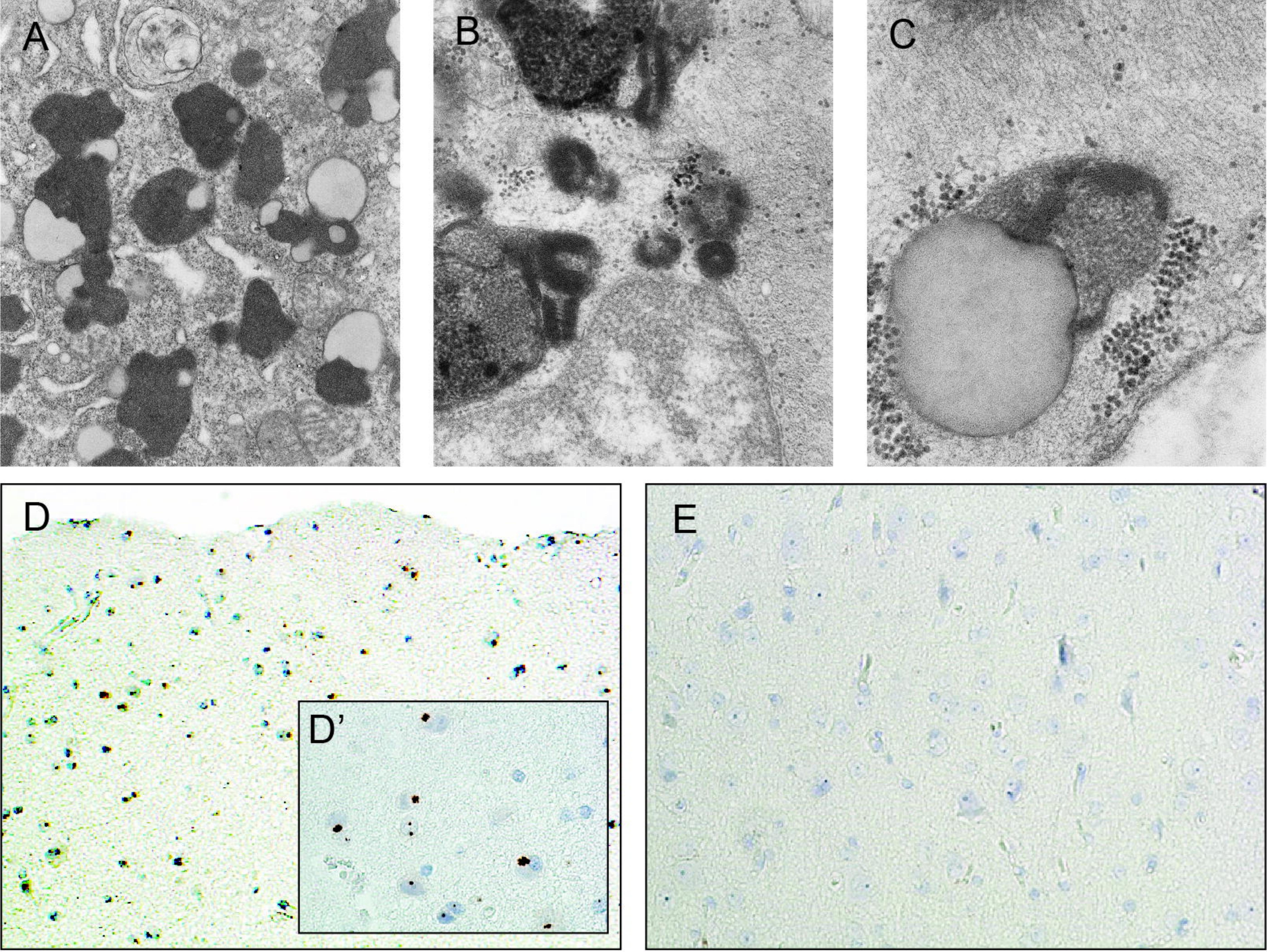



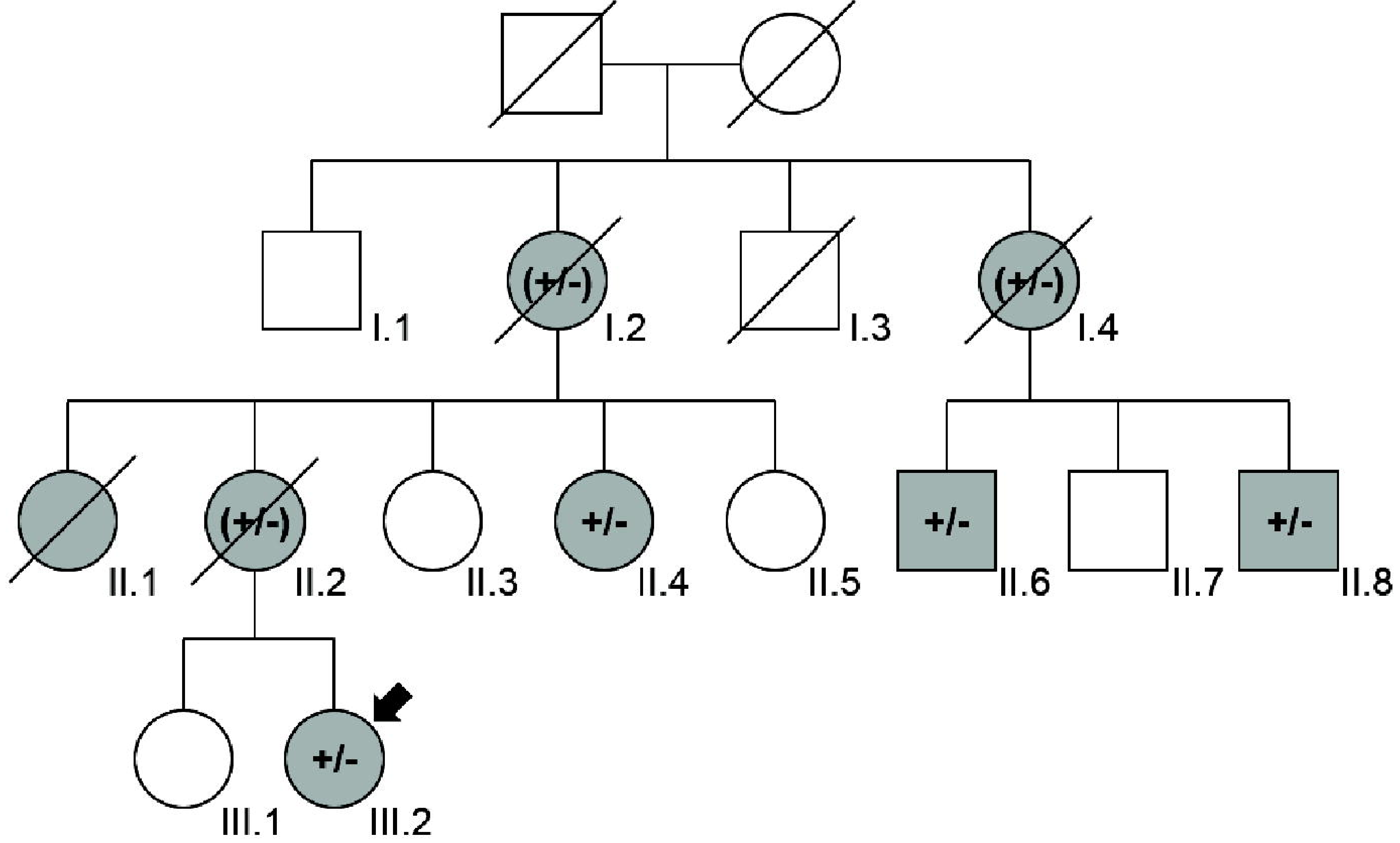

B

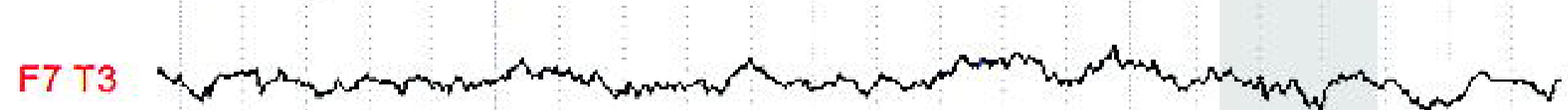

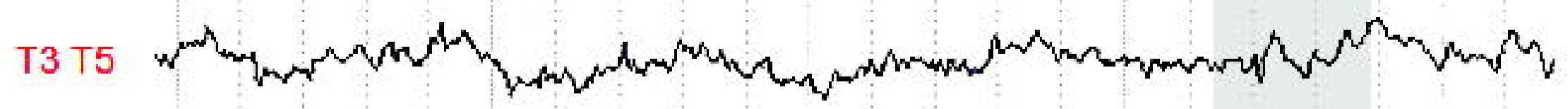

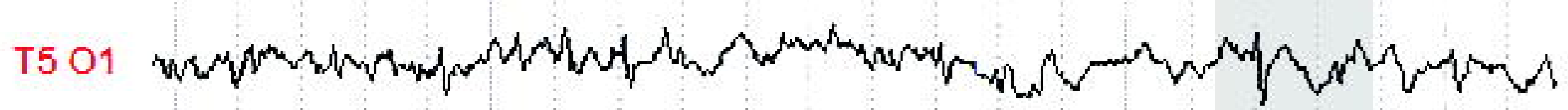

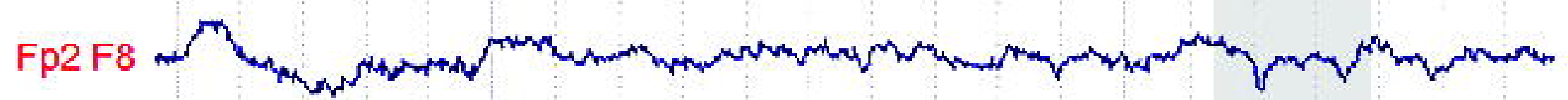

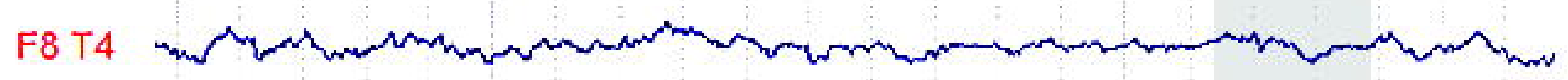

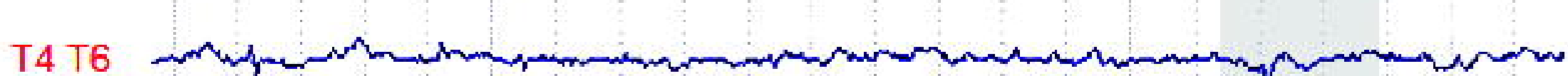

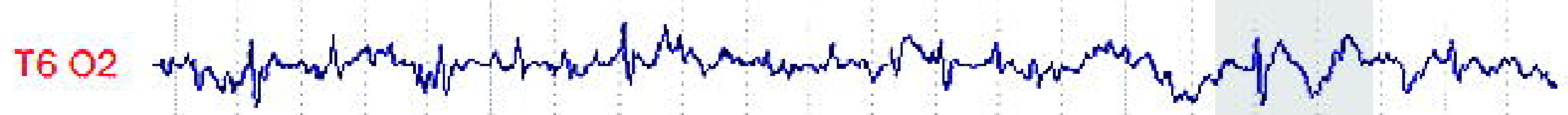

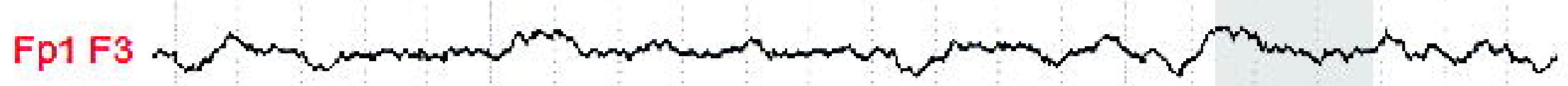

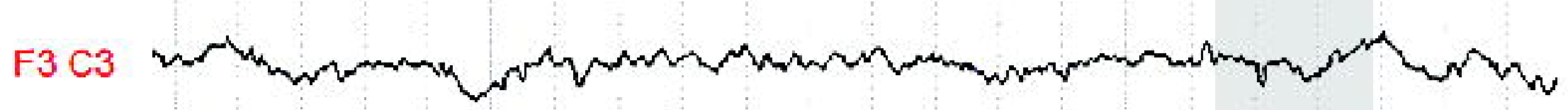

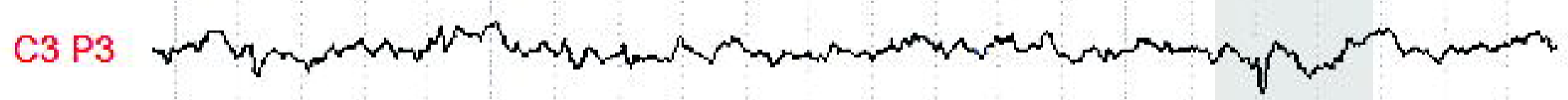

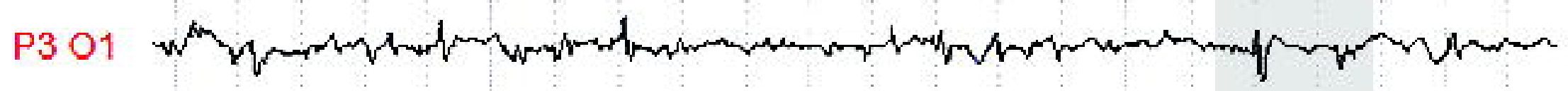

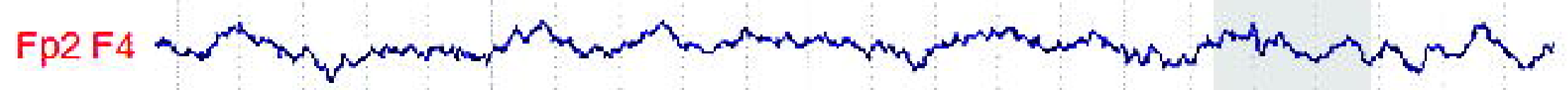

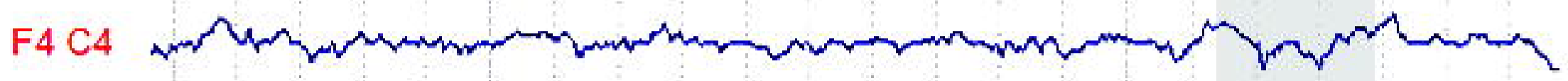

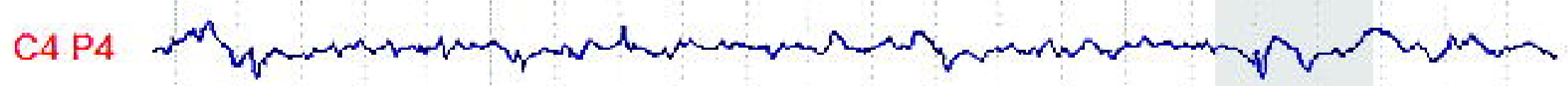

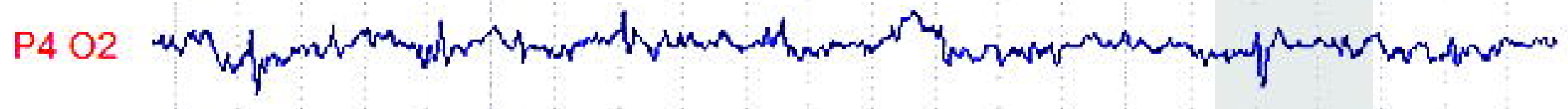

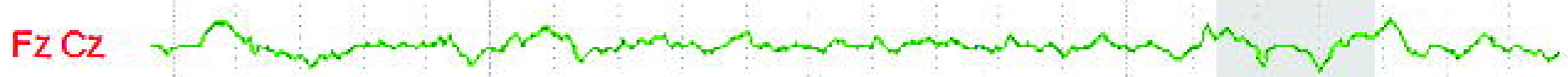

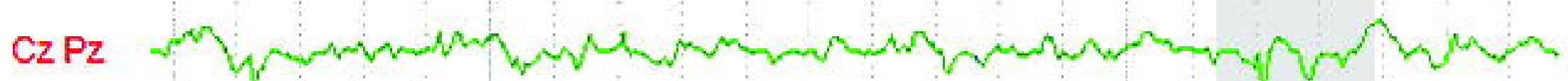

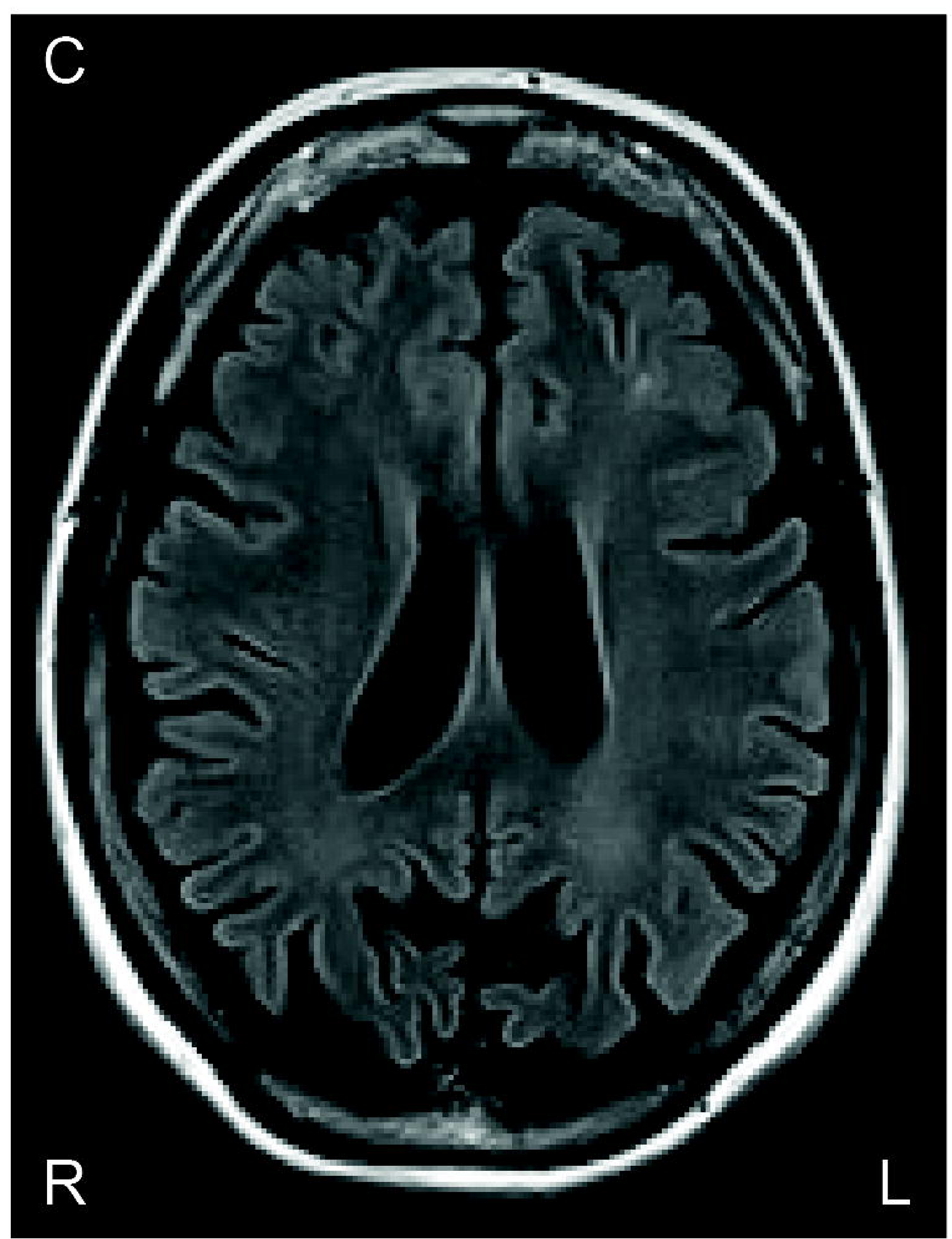

